\title{
Modificações da qualidade de vida sexual de obesos submetidos à cirurgia de Fobi-Capella
}

\section{Changes the sexual quality of life of the obeses submitted Fobi-Capella gastroplasty surgery}

Arakén Almeida de Araúuo'; Ana Maria de Brito2; Moacir de Novaes lima Ferreira3; Kátia Petribú4; Maria Helena de Araújo Mariano ${ }^{5}$

\section{R E S U M O}

\begin{abstract}
Objetivo: Analisar as modificações na qualidade de vida sexual de obesos, proporcionadas pela redução do peso corpóreo, após a realização da gastroplastia à Fobi-Capella. Método: Estudo quantitativo, prospectivo e longitudinal com cortes transversais, realizado entre março de 2005 e março de 2007, com 21 obesos mórbidos. Critérios de inclusão: idade de 20 a 50 anos, índice de massa corpórea (IMC) $=40 \mathrm{Kg} / \mathrm{m}^{2}$ e insucesso em tentativas de tratamento clínico para obesidade nos últimos dois anos. Coleta de dados clínicos em prontuários e aplicação de questionário específico com questões relativas à função sexual, realizadas antes e seis meses após a cirurgia. Os escores obtidos foram analisados com o auxilio do programa Epi-Info6, empregando o teste $\mathrm{T}$ de student para amostras pareadas. Resultados: $\mathrm{O}$ valor total aumentou seis meses após a cirurgia em $76,19 \%$, permaneceu inalterado em $14,29 \%$ e diminuiu em $9,52 \%$. Os domínios referentes a função erétil e a relação sexual aumentaram em $71,42 \%$, ao desejo sexual em $52,38 \%$ e ao orgasmo em $28,57 \%$. A satisfação sexual aumentou em $57,14 \%$. As médias do valor total e dos seus domínios antes e seis meses após a cirurgia, exceto aquelas referentes ao orgasmo e ao desejo sexual, apresentam diferenças estatisticamente significativas. Conclusão: A qualidade de vida sexual em homens obesos melhora após a realização da gastroplastia à Fobi-Capella. Evidenciaram-se modificações favoráveis na função sexual desses indivíduos após a perda de peso.
\end{abstract}

Descritores: Qualidade de vida. Sexualidade. Obesidade mórbida. Gastroplastia. Cirurgia bariátrica.

\section{INTRODUÇÃO}

A obesidade é considerada uma doença crônica, metabólica, de etiologia diversa que, segundo Sorensen'1, tem aumentado muito em incidência nas duas últimas décadas, levando a uma verdadeira epidemia que atinge a maioria dos países desenvolvidos e em desenvolvimento.

A obesidade Grau III (mórbida) está associada com o aumento da morbi-mortalidade por doença cardiovascular, diabetes, certos tipos de câncer, e a inadequação psicossocial decorrente de depressão e baixa autoestima ${ }^{2-4}$. Segundo Feldman et al. ${ }^{5}$, embora sua influência ainda não esteja bem esclarecida, se sabe que a obesidade interfere numa ampla variedade de funções, inclusive sexuais, independente de aspectos culturais.

No que concerne ao tratamento da obesidade, a tratamento cirúrgico é um procedimento que resulta em perda de peso significativo e duradouro, melhora as comorbidades, previne as complicações ameaçadoras da vida e aumenta a longevidade ${ }^{6}$. A derivação gastrojejunal em Y-de-Roux é considerada excelente opção técnica. Essa modalidade de tratamento cirúrgico é efetiva, confiável, apresenta baixos índices de re-intervenção e resulta em rápida perda de peso, podendo ocorrer redução acima de $50 \%$ do excesso de peso corpóreo 7,8 .

A sexualidade masculina, em parte, é uma expressão complexa que resulta do desejo, atração sexual e do comportamento sexual. Além da integridade anatômica e funcional dos órgãos genitais, a atividade sexual é influenciada por centros de excitação localizados no trato espinhal e no cérebro (sistema límbico), sendo que este último sofre efeitos significativos de fatores ambientais e sócioculturais. Características marcantes da sexualidade masculina, orientadas mais pelo tato e por estímulos visuais, podem ter como base as influências culturais e evolutivas através dos anos ${ }^{9}$. Do ponto de vista fisiológico, a função sexual masculina envolve a obtenção e manutenção da ereção peniana, a emissão espermática, a ejaculação e o orgasmo.

Para a maioria dos homens adultos, independente da massa corpórea, a função sexual é um aspecto importante da qualidade de vida. Todavia, homens portadores

Trabalho realizado nos Serviços de Cirurgia do Aparelho Digestivo do Hospital Universitário Oswaldo Cruz /UPE e do Hospital das Clínicas/UFPE. 1. Professor assistente do Departamento de Cirurgia da Faculdade de Ciências Médicas da Universidade de Pernambuco (FCM/UPE), PE, BR; 2. Professora adjunta do Departamento de Medicina Social da FCM/UPE, PE, BR; 3. Professor adjunto do Departamento de Medicina Clínica da FCM/UPE, PE, BR; 4. Professora Adjunta de Psiquiatria da FCM/UPE, PE, BR; 5. Médica Reumatologista do HUOC/UPE, PE, BR. 
de obesidade mórbida podem ter a qualidade de vida sexual afetada por disfunções sexuais que comprometem a ereção, a ejaculação, o orgasmo, e o desejo sexual, além de diversas limitações decorrentes da mobilidade e massa corporais, e da estigmatização social. Enfermidades frequentemente associadas à obesidade consideradas fatores de risco para disfunção erétil (DE), como o diabetes e as doenças cardiovasculares, alguns hábitos de vida como sedentarismo, alcoolismo e tabagismo, podem comprometer a função sexual masculina.

O objetivo deste estudo foi analisar a qualidade de vida sexual de uma amostra populacional de obesos mórbidos submetidos à operação para tratamento da obesidade, utilizando o IIEF (Quadro I) antes e seis meses após a realização da gastroplastia à Fobi-Capella, buscando esclarecer as modificações da função sexual decorrentes da perda de peso.

\section{MÉTODO}

Estudo quantitativo, prospectivo, longitudinal com dois cortes transversais inter-relacionados, desenvolvido no Hospital Universitário Oswaldo Cruz da Universidade de Pernambuco e no Hospital das Clínicas da Universidade Federal de Pernambuco, com um grupo de 21 homens portadores de obesidade Grau III, participantes do programa de cirurgia da obesidade e candidatos à gastroplastia à Fobi-Capella. Foram critérios de inclusão: idade de 20 a 50 anos, IMC=40 Kg/m², insucesso em tentativas de tratamento clínico para obesidade nos últimos dois anos e avaliação pré-operatória rigorosa (psicológica, nutricional, cardiológica, endocrinológica, pulmonar, gastro-enterológica, anestésica, laboratorial e por imagem).

O estudo foi realizado em duas etapas: na primeira, correspondente ao primeiro corte transversal, foi feita uma abordagem a partir de dados clínicos e laboratoriais obtidos durante a fase pré-operatória, em prontuários médicos dos serviços de cirurgia do aparelho digestivo dos Hospitais acima citados, entre março de 2005 e março de 2007. Após conhecimento e aceitação do termo de consentimento pós-informação, aplicou-se o questionário IIFF (Apêndice - páginas 47-48) em regime ambulatorial, um mês antes da operação. O referido questionário é composto por questões fechadas que foram respondidas pelo paciente sem interferência de terceiros ou do pesquisador. As questões estão divididas em cinco domínios de função sexual: função erétil (questões de 1 a 5 e 15), relação sexual (questões de 6,7 e 8), função orgásmica (questões 9 e 10), desejo sexual (questões 11 e 12), e satisfação sexual de modo geral (questões 13 e 14). Cada questão tem um valor que varia de 1 a 5 , sendo que respostas com valores baixos representam pobre condição da qualidade da vida sexual. Com base nos resultados relativos à função erétil, os graus de DE são definidos como leve (21 a 24), moderado (16 a 20) e grave (<15).
Na segunda etapa da pesquisa, correspondente ao segundo corte transversal, seis meses após a operação, aplicou-se novamente o questionário IIEF.

Para a análise estatística utilizou-se o teste $T$ de Student para amostras pareadas a fim de comparar as médias dos resultados antes e após a operação, com o auxílio do programa de computação Epi-Info6.

\section{RESULTADOS}

De março de 2005 a março de 2007, foram analisados 21 indivíduos com média de idade igual a 37 anos. Treze $(61,9 \%)$ hipertensos, um $(4,76 \%)$ diabético e três $(14,28 \%)$ portadores de ambas as enfermidades, todos em uso de medicamentos específicos. Quatro pacientes $(19,04 \%)$ não apresentavam sinais clínicos ou laboratoriais de doença em atividade. O resultado total do questionário IIEF aumentou num período de seis meses após a operação em $76,19 \%$, permaneceu inalterada em $14,29 \%$ e diminui em 9,52\% (Tabela 1). Os domínios referentes à função erétil e à relação sexual aumentaram em $71,42 \%$, ao desejo sexual em $52,38 \%$ e ao orgasmo em $28,57 \%$. A satisfação sexual de modo geral aumentou em 57,14\% (Tabela 2).

As médias do resultado total do questionário IIEF e dos seus domínios antes e seis meses após a realização do ato operatório exceto aquelas referentes ao orgasmo e ao desejo sexual, apresentam diferenças estatisticamente

Tabela 1 - Valor total do IIEF antes e seis meses após a realização do tratamento cirúrgico.

\begin{tabular}{ccc}
\hline Antes & Após & Diferença \\
\hline 58 & 54 & -4 \\
70 & 67 & -3 \\
61 & 61 & 0 \\
61 & 61 & 0 \\
58 & 58 & 0 \\
69 & 70 & 1 \\
73 & 74 & 1 \\
59 & 61 & 2 \\
65 & 70 & 5 \\
55 & 60 & 5 \\
67 & 73 & 6 \\
66 & 72 & 6 \\
66 & 73 & 7 \\
60 & 67 & 7 \\
54 & 65 & 11 \\
54 & 66 & 12 \\
53 & 74 & 13 \\
61 & 69 & 8 \\
55 & 69 & 14 \\
45 & 61 & 16 \\
28 & 59 & 31 \\
\hline
\end{tabular}

IIEF: International index erectile function. 
Tabela 2 - Médias dos valores dos domínios do IIEF antes e seis meses após a cirugia.

\begin{tabular}{|c|c|c|c|c|c|}
\hline Domínios & & Média & $95 \%$ intervalo & de confiança & $p$ valor \\
\hline \multirow[t]{2}{*}{ Função erétil } & Antes & $24,00+/-5,99$ & 21,27 & 26,72 & 0,005 \\
\hline & Após & $27,85+/-2,45$ & 26,73 & 28,97 & \\
\hline \multirow[t]{2}{*}{ Relação sexual } & Antes & $10,09+/-2,58$ & 8,91 & 11,27 & 0,005 \\
\hline & Após & $11,80+/-2,20$ & 10,80 & 12,81 & \\
\hline \multirow[t]{2}{*}{ Orgasmo } & Antes & $9,14+/-1,27$ & 8,56 & 9,72 & 0,234 \\
\hline & Após & $9,38+/-0,97$ & 8,93 & 9,82 & \\
\hline \multirow[t]{2}{*}{ Desejo sexual } & Antes & $7,66+/-2,03$ & 6,74 & 8,59 & 0,061 \\
\hline & Após & $8,61+/-1,32$ & 8,01 & 9,22 & \\
\hline \multirow[t]{2}{*}{ Satisfação sexual } & Antes & $7,09+/-1,48$ & 6,25 & 7,93 & 0,031 \\
\hline & Após & $8,09+/-1,13$ & 7,57 & 8,61 & \\
\hline
\end{tabular}

IIEF: International index erectile function.

Tabela 3 - Médias dos valores do IIEF antes e seis meses após a operação.

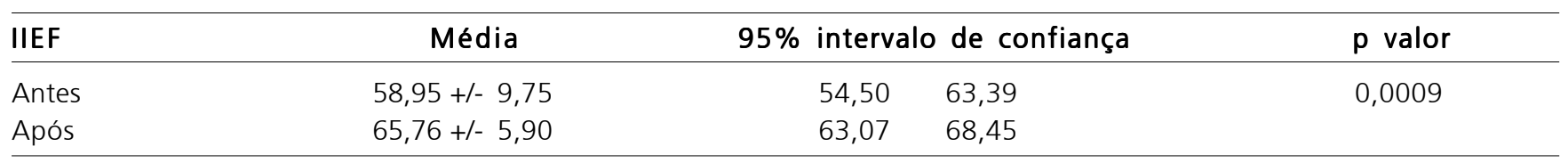

IIEF: International index erectile function.

significativas. Salientamos, no entanto, que os resultados referentes ao desejo sexual apresentaram tendência a significância estatística. (Tabelas 2 e 3).

Em seguimento clínico-ambulatorial seis meses após a realização do ato operatório, 18 participantes $(85,71 \%)$ relataram que ocorreram modificações favoráveis da atividade sexual, enfatizando melhora acentuada da relação sexual de forma geral, do orgasmo e do desejo sexual.

\section{DISCUSSÃO}

As doenças crônicas mais freqüentemente associadas à obesidade, como o diabetes e a hipertensão arterial sistêmica e os seus respectivos tratamentos, além de comprometerem a função sexual masculina, são causas relevantes de complicações sistêmicas e de óbito. Feldman et al. ${ }^{5}$ não demonstraram correlação entre obesidade e DE na população geral, no entanto, ressaltam que a associação de várias doenças à obesidade, como diabetes melitus, hipertensão arterial, dislipidemia, tireopatias, dentre outras, pode estar relacionada aos distúrbios da ereção. Para avaliar objetivamente o impacto da obesidade na função erétil, Chung et al. ${ }^{10}$ aferiram a capacidade erétil de 321 homens portadores de DE por meio de ultrassom doppler das artérias cavernosas do pênis. Concluíram os autores que, a qualidade de função erétil residual foi melhor em pacientes não-obesos que obesos, a incidência de fatores de risco vasculares foi mais alta em pacientes obesos e, quando esses fatores foram excluídos não houve diferença estatisticamente significativa considerando função erétil residual ou a incidência de dano vascular peniano entre os pacien- tes obesos e não-obesos. Por fim, enfatizam que a obesidade por si só, parece não ser um fator fundamental e independente para função erétil, mas pelo menos contribui como um fator mediador para aumentar o risco de o paciente ter doenças associadas que são fatores de riscos para DE. Em nosso meio, Abdo et al. ${ }^{11}$, apresentaram um perfil da vida sexual da população brasileira, mostrando que 6,8\% dos entrevistados com DE referiam ser obesos contra apenas 3,3\%, sem $D E$, que referiam ser obesos.

Para compreender a função sexual de homens obesos, deve-se ter em mente que a DE está associada a várias formas de doenças sistêmicas relacionadas à obesidade e também às alterações comportamentais ou psicossocias. Além da possibilidade de alterar o bem estar emocional e interferir profundamente na autoestima do homem comprometendo sua função sexual, a DE quando presente nos obesos repercute negativamente na qualidade de vida.

Estudo randomizado e multicêntrico com aproximadamente 28.000 entrevistados, mostrou que boa saúde é um dos fatores significativamente importantes para a qualidade de vida, assim como autoestima é um fator determinante para a masculinidade ${ }^{12}$.

Diante do comprovado aumento da prevalência da obesidade e dos índices elevados de prevalência da DE, notadamente em homens portadores de fatores de risco frequentemente associados à obesidade, estudos sobre a qualidade de vida sexual que avaliem os parâmetros relevantes da função sexual, como função erétil, relação sexual, função orgásmica, desejo sexual e satisfação sexual de modo geral, são necessários. Vários estudos mostram significativa melhora na qualidade de vida após a realização de procedimentos cirúrgicos do tipo gastroplastia reduto- 
$r^{4,13-15}$. Entretanto, inexistem estudos sobre os efeitos das diversas modalidades de cirurgia bariátrica, inclusive da gastroplastia à Fobi-Capella, sobre a qualidade de vida sexual.

Na elaboração de um estudo ou de uma determinada avaliação para mensuração da qualidade de vida relacionada à saúde, são utilizados instrumentos ou questionários. O instrumento pode ser genérico ou específico, o primeiro inclui grande parte das dimensões que compõem a qualidade de vida, sendo útil na condução de pesquisas gerais sobre saúde e para comparar estados de doença, e à medida que se concentra em um aspecto em particular, em uma determinada doença e ou tratamento, é chamado específico ${ }^{16}$. Nos últimos 20 anos, pesquisadores têm demonstrado interesse em usar instrumentos de avaliação padronizados para avaliar o efeito da obesidade na qualidade de vida.

O questionário International Index Erectile Function (IIEF) é um instrumento específico que avalia os parâmetros relevantes e, os resultados de um tratamento baseado na percepção do próprio individuo sobre a sua vida sexual ${ }^{17}$. É confiável e auto-aplicável para avaliação da função erétil, e foi validado no Brasil em 1998 por Ferraz \& Ciconelli18.

Veronelli et al. ${ }^{19}$ utilizando o questionário IIEF, mostraram que num grupo de homens com idade entre 23 a 50 anos, o índice de DE foi pior em pacientes obesos que em pacientes controle e melhor que em pacientes com tireopatia. Os pacientes diabéticos apresentaram índices de DE piores que os pacientes controle. Assim, pode-se inferir que pacientes obesos são particularmente de risco para DE na faixa etária sexualmente mais ativa.

Em estudo utilizando o questionário IIEF, Espósito et al. ${ }^{20}$ mostraram melhora significativa da DE em homens obesos mórbidos com DE grave, porém, sem co-morbidades sistêmicas, dois anos após tratamento com modificações no estilo de vida que incluiu redução da ingestão calórica e aumento da atividade física, ressaltando que esta conduta resultou também na melhora da função endotelial e dos marcadores inflamatórios. Esse estudo demonstra a importância do questionário IIEF para a avaliação da DE durante tratamento para a obesidade, o que nos permite assegurar a sua utilização em pesquisas com homens obesos mórbidos candidatos e submetidos à gastroplastia à Fobi-Capella.

Para análise da qualidade de vida sexual em homens portadores de obesidade grau III, o questionário IIEF pode ser aplicado antes e após a realização de gastroplastia à Fobi-Capella.

No presente estudo, as respostas analisadas através do questionário IIEF seis meses após a realização da gastroplastia evidenciam melhora considerável da qualidade de vida sexual, manifestada por aumento da média dos valores relativos a todos os domínios de função sexual, destacando-se aquelas que apresentam diferença estatisticamente significativa, relativas a função erétil, a relação sexual e a satisfação sexual de modo geral. Seis $(28,5 \%)$ pacientes antes da gastroplastia eram portadores de DE: grave (dois), moderada (um) e leve (três). Todos obtiveram aumento dos valores do IIEF seis meses após a realização da gastroplastia, sendo que quatro apresentaram pontuação do $\| \mathrm{EF}>25$, que representa função erétil normal, e aqueles que eram portadores de DE grave tornaram-se portadores de DE leve (IIEF 21 a 24). Esses achados são importantes porque representam uma melhora clínica considerável e significativa da função sexual e, não apenas dados estatísticos significativos. As diferenças não estatisticamente significativas relativas ao orgasmo e ao desejo sexual, ratificam o conceito psicológico desses domínios da função sexual, o que talvez requeira pesquisas especificas para melhor esclarecimento destes domínios. Aproximadamente $62 \%$ e mais de $4 \%$ dos sujeitos do grupo estudado, eram hipertensos e diabéticos, respectivamente, em uso de medicações especificas, antes de se submeterem à gastroplastia. Apresentavam IMC em torno de $63 \mathrm{Kg} / \mathrm{m}^{2}$. Situação não evidenciada seis meses após a realização do procedimento cirúrgico, quando se constatou a ausência desses fatores de risco importantes para DE e consequentemente o uso de medicações especificas, redução do peso corpóreo observado antes da realização da operação em torno de 30\% e mudanças favoráveis da qualidade de vida sexual. A gastroplastia à Fobi-Capella proporciona em menor espaço de tempo prevenção das complicações provocadas pelas doenças acima citadas, redução dos fatores de riscos para DE e, pode contribuir para a prevenção da DE em pacientes obesos mórbidos.

Os resultados deste trabalho são consistentes com os estudos publicados sobre qualidade de vida que utilizaram instrumentos de avaliação diversos, diferentes do questionário IIEF ${ }^{4,13-14}$. Sugerem que o conhecimento prévio das modificações favoráveis da função sexual, proporcionadas pela operação, pode facilitar a aceitação e confiança na tomada de decisão para o tratamento cirúrgico.

A falta de estudos que relatem os efeitos na qualidade de vida sexual após este tipo de operação, utilizando um questionário especifico composto de domínios sobre a função sexual, representa uma importante lacuna que requer pesquisas nessa área. A disfunção sexual pode ser um problema freqüente em obesos mórbidos. Suas manifestações clínicas são quase sempre omitidas por estes indivíduos e, ao mesmo tempo, pouco interrogadas pelos profissionais de saúde que integram equipes multidisciplinares para o tratamento cirúrgico da obesidade. Diante disso, recomenda-se que questões relativas à sexualidade sejam consideradas na avaliação e preparação pré-operatória de pacientes candidatos ao tratamento cirúrgico.

Apesar do pequeno número de participantes do grupo estudado, são expressivos os dados estatísticos. Isso denota que, pesquisas sobre a qualidade de vida sexual de pacientes obesos candidatos a gastroplastia à Fobi-Capella devem ser incentivadas e os seus resultados amplamente divulgados por equipes multidisciplinares que planejam e preparam pacientes obesos mórbidos para tratamento cirúrgico.

Até a presente data não há relato na literatura sobre a utilização do questionário IIEF, para análise da fun- 
ção sexual de homens obesos submetidos a algum tipo de cirurgia bariátrica.

Os resultados acima permitem inferir que, a redução do peso corpóreo proporcionado pela gastroplastia à Fobi-Capella melhora a qualidade de vida sexual, seis meses após o procedimento cirúrgico.
Com base nesses dados, depreende-se que homens portadores de obesidade grau III submetidos à gastroplastia à Fobi-Capella apresentam modificações favoráveis que proporcionam melhora da função sexual e, por conseguinte, da qualidade de vida.

\title{
A B S T R A C T
}

\begin{abstract}
Objectives: Examine changes in the quality of sexual life of men with morbid obesity offered by a reduction in body weight following Fobi-Capella gastroplasty. Method: A quantitative, prospective, longitudinal, cross-sectional study was carried out on 21 men with morbid obesity between March 2005 and March 2007. Inclusion criteria were: aged 20 to 50 years, body mass index (BMI) $=40 \mathrm{~kg} / \mathrm{m}^{2}$ and failure in attempts of clinical treatment for obesity in the previous two years. Data collection from clinical records and the administering of a specific questionnaire on issues relating to sexual function was performed prior to and six months following surgery. Scores were analyzed with the help of the Epi-Info 6 program, using the Student's t-test for paired samples. Results: The total score increased at the six-month post operative in 76.19\%; remained unaltered in 14.29\%; and decreased in 9.52\%. Scores in areas related to erectile function and sexual relations increased in $71.42 \%$; sexual desire increased in $52.38 \%$; and orgasms increased in $28.57 \%$. General sexual satisfaction increased in $57.14 \%$. The average total score and scores on the domains of the questionnaire prior to and six months following surgery revealed statistically significant differences, except those relating to sexual desire and orgasm. Conclusion: The quality of sexual life in morbid obese men improves following Fobi-Capella surgery. Favorable changes occurred in sexual function after these individuals underwent this type of bariatric surgery.
\end{abstract}

Key Words: Quality of life. Sexuality. Obesity morbid. Gastroplasty. Bariatric surgery.

\section{REFERENCIAS}

1. Sorensen TI. The changing lifestyle in the world. Body weight and what else? Diabetes Care. 2000; 23 (1 Suppl2):B1-4.

2. Pi-Sunyer FX. Medical hazards of obesity. Ann Intern Med. 1993; 119(7 Pt 2):655-60.

3. Kopelman PG. Obesity as a medical problem. Nature. 2000; 404(6778):635-43.

4. Dymek MP, Le Grange D, Neven K, Alverdy J. Quality of life after gastric bypass surgery: a cross-sectional study. Obes Res. 2002; (10):1135-42.

5. Feldman HA, Goldstein I, Hatzchristou DG, Krane RJ, McKinlay JB. Impotence and its medical and psychological correlates: results of the Massachussets Male Ageing Study. J Urol. 1994; 151(1):54-61.

6. Cowan GS Jr., Hiler ML, Buffington C. Criteria for selection of patients for bariatric surgery. Eur J Gastroenterol. 1999; 11(2):69-75.

7. Garrido $A B$ Jr. Cirurgia em obesos mórbidos: experiência pessoal. Arq Bras Endocrinol Metabol. 2000; 44(2):106-13.

8. Pories WJ, Roth JS. Gastric bypass. In: Martin LF, editor. Obesity surgery. New York: McGraw-Hill; 2004. p. 213-26.

9. Joseph R. The evolution of sex differences in language, sexuality, and visual-spatial skills. Arch Sex Behav. 2000; 29(1):35-66.

10. Chung WS, Sohn JH, Park YY. Is obesity an underlyng factor in erectile dysfunction? Eur Urol. 1999; 36(1):68-70

11. Abdo CH, Oliveira WM Jr, Moreira Jr, Fittipaldi JA. Perfil sexual da população brasileira: resultado do Estudo do Comportamento Sexual do Brasileiro (ECOS) do brasileiro. Rev Bras Med. 2002; 59(4):250-7.

12. Sand MS, Fisher W, Rosen R, Heiman J, Eardley I. Erectile dysfunction and constructs of masculinity and quality of life in the multinational Men's Attitudes to Life Events and Sexuality (MALES) study. I Sex Med. 2008; 5(3):583-94. Epub 2008 Jan 21.

13. Kolotkin RL, Binks M, Croby RD, Østbye T, Gress RE, Adams TD. Obesity and sexual quality of life. Obesity. 2006; 14(3):472-9.

14. Barreto Villela NB, Braghrolli Neto O, Lima Curvello K, Eduarda Paneili B, Seal C, Santos D, Cruz T. Quality of life of obese patients submitted to bariatric surgery. Nutr Hosp. 2004; 19(6):367-71.
15. Herpertz S, Kielmann R, Wolf AM, Langkafel M, Senf W, Hebebrand J. Does obesity surgery improve psychosocial functioning? A systematic review. Int J Obes Relat Metab Disord. 2003; 27(11):1300-14.

16. Guyatt GH, Feeny DH, Parick DL. Measuring health- related quality of life. Ann Intern Med. 1993; 118(8):622-29.

17. Rosen RC, Cappelleri JC, Gendrano N $3^{\text {rd }}$. The international Index of Erectile Function (IIEF): a state-of-the-science review. Int J Impot Res. 2002; 14(4): 226-44.

18. Ferraz MB, Ciconelli M. Tradução e adaptação cultural do índice internacional de função erétil para a língua portuguesa. Rev Bras Med. 1998; 55(1):35-40.

19. Veronelli A, Masu A, Ranieri R, Rognoni C, Laneri M, Pontiroli AE. A Prevalence of erectile dysfunction in thyroid disorders: comparison with control subjects and with obese and diabetic patients. Int J Impot Res. 2006; 18(1):111-4.

20. Esposito K, Giugliano F, Di Palo O, Giugliano G, Marfella R, D'Andrea F, D'Armiento M, Giugliano D. Effect of lifestyle changes on erectile dysfunction in obese men: a randomized controlled trial. JAMA. 2004; 291(24):2978-84.

Recebido em 08/08/2008

Aceito para publicação em 08/10/2008

Conflito de interesses: nenhum

Fonte de financiamento: nenhuma

\section{Como citar este artigo:}

Araújo AA, Brito AM, Ferreira MNL, Petribú K, Mariano MHA. Modificações da qualidade de vida sexual de obesos submetidos à cirurgia de Fobi-Capella. Rev Col Bras Cir. [periódico na Internet] 2009; 36(1). Disponível em URL: http://www.scielo.br/rcbc

\section{Endereço para correspondência:}

Arakén Almeida de Araújo

E-mail: arakenaa@elogica.com.br 
Apêndice - IIEF (International index erectile function).

Perguntas referentes à ereção

1. Com que frequência você consegue uma ereção durante a atividade sexual?

1 - Quase nunca/nunca

2 - Poucas vezes (muito menos que a metade das vezes)

3 - Algumas vezes (aproximadamente metade das vezes)

4 - Na maioria das vezes (muito mais que a metade das vezes)

5 - Quase sempre/sempre

2. Quando você tem ereções após estímulo sexual, com que frequência suas ereções são suficientemente rígidas para a penetração?

0 - Nenhuma atividade sexual

1 - Quase nunca/nunca

2 - Poucas vezes (muito menos que a metade das vezes)

3 - Algumas vezes (aproximadamente metade das vezes)

4 - Na maioria das vezes (muito mais que a metade das vezes)

5 - Quase sempre/sempre

3. Quando você tentou ter uma relação sexual, com que frequência você conseguiu penetrar sua companheira?

1 - Quase nunca/nunca

2 - Poucas vezes (muito menos que a metade das vezes)

3 - Algumas vezes (aproximadamente metade das vezes)

4 - Na maioria das vezes (muito mais que a metade das vezes)

5 - Quase sempre/sempre

4. Durante a relação sexual, com que frequência você consegue manter a ereção depois de ter penetrado sua companheira?

0 - Não tentei ter relação sexual

1 - Quase nunca/nunca

2 - Poucas vezes (muito menos que a metade das vezes)

3 - Algumas vezes (aproximadamente metade das vezes)

4 - Na maioria das vezes (muito mais que a metade das vezes)

5 - Quase sempre/sempre

5. Durante a relação sexual, qual seu grau de dificuldade para manter a ereção até completar a relação sexual?

0 - Não tentei ter relação sexual

1 - Extremamente difícil

2 - Muito difícil

3 - Difícil

4 - Um pouco difícil

5 - Não é difícil

\section{Perguntas referentes à satisfação com a relação sexual}

6. Quantas vezes você tentou ter relação sexual?

0 - Não tentei

1 - Uma a duas tentativas

2 - Três a quatro tentativas

3 - Cinco a seis tentativas

4 - Sete a dez tentativas

5 - Onze ou mais tentativas

7. Quando você tentou ter relações sexuais, com que frequência foi satistatória para você?

0 - Não tentei ter relação sexual

1 - Quase nunca/nunca

2 - Poucas vezes (muito menos que a metade das vezes)

3 - Algumas vezes (aproximadamente metade das vezes)

4 - Na maioria das vezes (muito mais que a metade das vezes)

5 - Quase sempre/sempre

8. Qual o seu grau de prazer na relação sexual?

0 - Nenhuma atividade sexual

1 - Nenhum prazer

2 - Não muito prazerosa

3 - Razoavelmente prazerosa

4 - Muito prazerosa

5 - Extremamente prazerosa 


\section{Perguntas referentes à ejaculação}

9. Quando você foi sexualmente estimulado ou teve uma relação sexual, com que frequência ejaculou?

1 - Quase nunca/nunca

2 - Poucas vezes (muito menos que a metade das vezes)

3 - Algumas vezes (aproximadamente metade das vezes)

4 - Na maioria das vezes (muito mais que a metade das vezes)

5 - Quase sempre/sempre

10. Quando você foi sexualmente estimulado ou teve uma relação sexual, com que frequência você teve a sensação de orgasmo ou clímax?

0 - Sem estimulação sexual

1 - Quase nunca/nunca

2 - Poucas vezes (muito menos que a metade das vezes)

3 - Algumas vezes (aproximadamente metade das vezes)

4 - Na maioria das vezes (muito mais que a metade das vezes)

5 - Quase sempre/sempre

\section{Perguntas referentes ao desejo sexual}

11. Com que frequência você sentiu desejo sexual?

1 - Quase nunca/nunca

2 - Poucas vezes (muito menos que a metade das vezes)

3 - Algumas vezes (aproximadamente metade das vezes)

4 - Na maioria das vezes (muito mais que a metade das vezes)

5 - Quase sempre/sempre

12. Como você classificaria seu nível de desejo sexual?

1 - Muito baixo/nenhum

2 - Baixo

3 - Moderado

4 - Alto

5 - Muito alto

\section{Perguntas referentes à satisfação sexual de modo geral}

13. Qual seu grau de satisfação com sua vida sexual de maneira geral?

1 - Muito baixo

2 - Baixo

3 - Moderado

4 - Alto

5 - Muito alto

14. Qual seu grau de satisfação com o relacionamento sexual com sua companheira?

1 - Muito insatisfeito

2 - Moderadamente insatisfeito

3 - Nem satisfeito, nem insatisfeito

4 - Moderadamente satisfeito

5 - Muito satisfeito

15. Qual seu grau de confiança de que você pode conseguir e manter uma ereção?

1 - Muito baixo

2 - Baixo

3 - Moderado

4 - Alto

5 - Muito alto 